\section{ORIGINAL RESEARCH}

\author{
R.J. McDonald \\ J.S. McDonald \\ J.P. Bida \\ D.F. Kallmes \\ H.J. Cloft

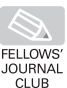

\title{
Subarachnoid Hemorrhage Incidence in the United States Does Not Vary with Season or Temperature
}

BACKGROUND AND PURPOSE: Previous studies have suggested seasonal variations in rates of spontaneous rupture of intracranial aneurysms, leading to potentially devastating $\mathrm{SAH}$. In an effort to identify a seasonal effect, variation in SAH incidence and in-hospital mortality rates were examined as they relate to admission month, temperature, and climate using HCUP's Nationwide Inpatient Sample.

\begin{abstract}
MATERIALS AND METHODS: Cases of nontraumatic SAH and subsequent in-hospital mortality were extracted from the 2001-2008 NIS and associated with month of occurrence, local average monthly temperatures, and USDA climate zone. Multivariate regression analysis was used to study how admission month, temperature, and climate affected SAH admission and mortality rates.
\end{abstract}

RESULTS: Among 57,663,486 hospital admissions from the 2001-2008 NIS, 52,379 cases of spontaneous SAH (ICD-9-CM 430) and 13,272 cases of subsequent in-hospital mortality were identified. SAH incidence and in-hospital mortality rates were not significantly correlated with a monthly/seasonal effect (incidence, $\chi^{2}=2.94, P=.99$; mortality, $\chi^{2}=6.91, P=.81$ ). However, SAH incidence significantly varied with climate $(P<.0001$, zones 11 and 7$)$ but not with temperature $(P=.1453)$, whereas average monthly temperature and climate had no significant correlation with in-hospital mortality (temperature, $P=.3005$; climate, $P=.0863$ ).

CONCLUSIONS: We identified no significant monthly or temperature-related effect in the incidence of $\mathrm{SAH}$. Our data suggest that certain climate zones within the United States may be associated with significantly different SAH incidence, but the origins of these differences remain unclear and are probably unrelated to meteorologic variables.

ABBREVIATIONS: HCUP $=$ Healthcare Cost and Utilization Project; NIS $=$ Nationwide Inpatient Sample; $R E M L=$ restricted maximum likelihood; $R R=$ relative rate; $U S D A=U . S$. Department of Agriculture; VIF = variance inflation factor

$\mathbf{T}$ he incidence of SAH has been widely reported to vary seasonally due to changes in temperature, barometric pressure, and other weather-related phenomena. ${ }^{1-25}$ Circadian and seasonal variations in blood pressure, infection frequency, ethanol and tobacco use, and sedentary behavior, in addition to alteration in blood pressure after strenuous exertion in colder climates, have all been proposed as mechanisms to explain seasonal variation in SAH. ${ }^{26-39}$ Despite these well-established effects, the seasonal effects reported by some groups have not always been consistent with respect to timing, and other studies have failed to demonstrate any seasonal relationship. ${ }^{23,24,37-42}$ Thus, there remains no consensus regarding the influence of season and/or weather on the incidence rate of SAH. To adequately address this question of seasonal variation, a data base including a large number of hospital admissions is necessary. The HCUP's Nationwide Inpatient Sample is such a data base and represents approximately $20 \%$ of the yearly nonfederal hospitalizations in the United States. As the NIS is currently collected from 42 states, it is possible to sample the entire range of climates found within the complex geo-

Received May 17, 2011; accepted after revision January 6, 2012.

From the Clinician Investigator Training Program (R.J.M.), Department of Radiology (R.J.M., J.S.M., D.F.K., H.J.C.), Department of Laboratory Medicine and Pathology (J.S.M.), Department of Neurosurgery (D.F.K., H.J.C.), and Mayo Graduate School, College of Medicine (J.P.B.), Mayo Clinic, Rochester, Minnesota.

Please address correspondence to Robert J. McDonald, MD, PhD, Department of Radiology, Mayo Clinic, 200 1st St SW, Rochester, MN 55905; e-mail: mcdonald.robert@mayo.edu http://dx.doi.org/10.3174/ajnr.A3059 graphic landscape of the United States. Because climate regions are uniquely defined by meteorologic characteristics proposed to play a role in the seasonal incidence of SAH (temperature, atmospheric pressure, wind, rainfall, and particulate counts), we incorporated climate data into the NIS dataset to determine whether seasonal and/or meteorologic factors, including month, temperature, and/or climate zone, impact the incidence of SAH admissions and in-hospital mortality.

\section{Materials and Methods}

\section{Data Acquisition}

All SAH admissions (ICD-9-CM code 430) records were extracted from the 2001-2008 NIS hospital discharge data base (HCUP, Agency for Healthcare Research and Quality, Rockville, Maryland). ${ }^{43}$ Traumatic events (ICD-9-CM 800-805, 958.2) associated with SAH hospitalizations were excluded to avoid confounding effects. For all records meeting these inclusion and exclusion criteria, admission (year, month, and state), patient (age, sex, mortality during hospitalization), and hospital (hospital bed size [large, medium, small], teaching status [teaching versus nonteaching], address, zip code, location [urban versus rural], and NIS hospital identifier code) variables were extracted from the 2000-2008 NIS dataset. ${ }^{44}$ Patient age was divided into 10-year interval strata ranging from $1(0-10$ years of age $)$ to 11 $(>100$ years of age). Average monthly temperatures were acquired from the National Oceanographic and Atmospheric Administration for each unique hospital ID zip code and stratified in 10-degree incre- 


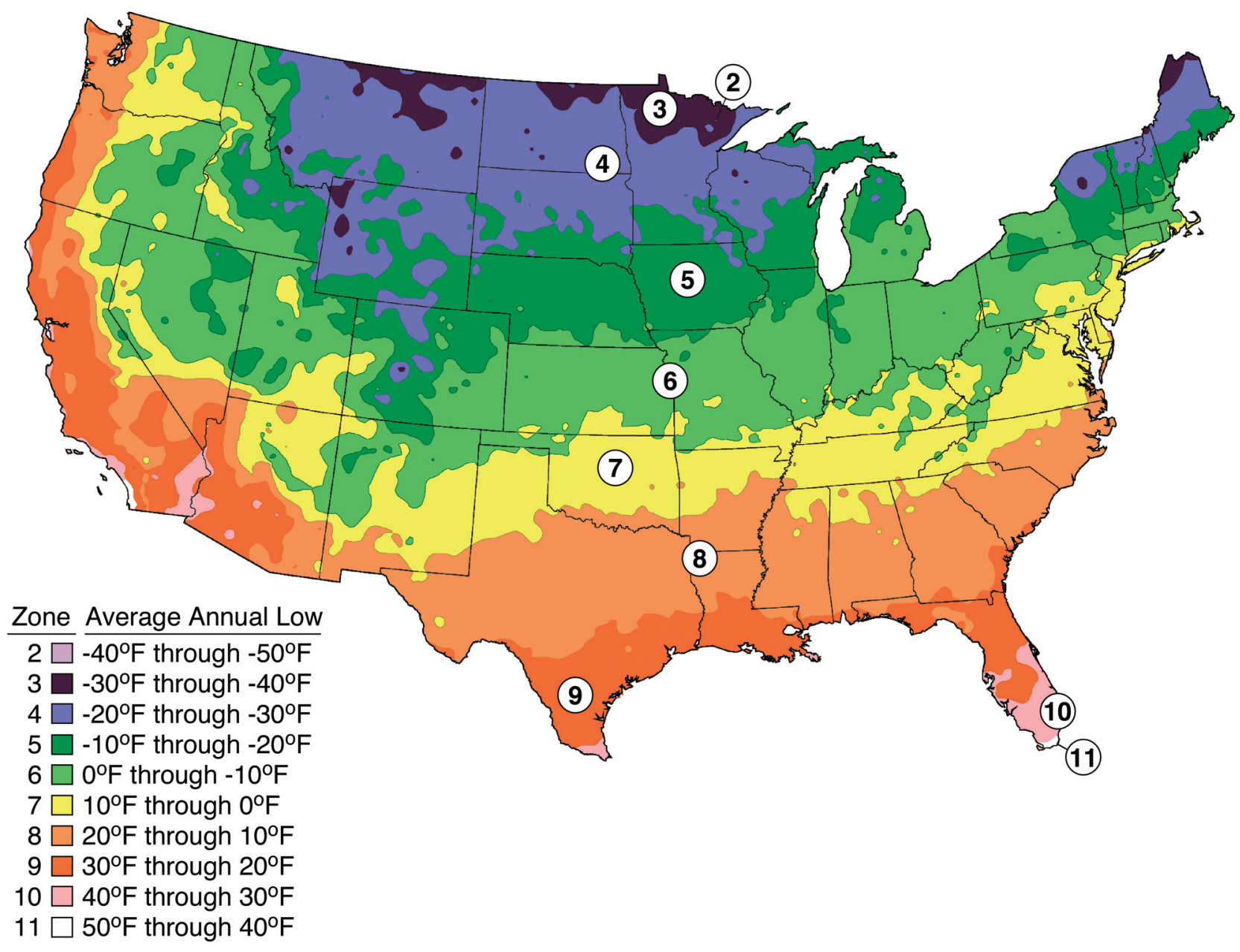

Fig 1. USDA hardiness zone map. Used with permission from the Arbor Day Foundation (http://www.arborday.org/media/zones.cfm).

ments $\left(<30^{\circ} \mathrm{F}, 30-39^{\circ} \mathrm{F}, 40-49^{\circ} \mathrm{F}, 50-59^{\circ} \mathrm{F}, 60-69^{\circ} \mathrm{F}, 70-79^{\circ} \mathrm{F}\right.$, $\left.>80^{\circ} \mathrm{F}\right) .{ }^{45}$ In addition, the local climate of each hospital was determined using USDA plant hardiness zone definitions. These discrete zones, originally conceived by the USDA to better delineate vegetation zones, divide the North American continent and Hawaii into 11 regions based on $10^{\circ} \mathrm{F}$ intervals in the average annual minimum temperatures and serve as a surrogate of the various climates that exist within the United States (Fig 1). These data, obtained from the USDA, were assigned to the NIS data by matching individual hospital ID zip codes to their respective hardiness zone, and were subdivided into USDA zones 3-11. ${ }^{46-48}$ Zones 1 and 2 were not included in this analysis, as they represent climate zones/geographic areas that lack hospitals participating in the NIS or represent regions outside of the United States. As these climate zones define the unique seasonal patterns in temperature and other meteorologic variables, they provide a complementary means of investigating the effects of season and climate on the incidence of SAH. Admission counts of SAH, in-hospital mortality counts, and total admission counts were determined for each combination of admission month, hospital ID, and USDA zone, average monthly temperature, age stratum, and sex.

\section{Statistical Analysis}

All extraction and manipulation of NIS data and subsequent statistical analyses were performed using commercial software (JMP, ver- sion 9; SAS, version 9.3; SAS Institute, Cary, North Carolina). Predictors of SAH incidence and SAH in-hospital mortality incidence were identified with Poisson-distributed generalized linear regression models, using a log-linkage function offset by the log of total admission counts for each month to control for differences in admission rates between hospitals. Predictor variables included in these models were admission month, average monthly temperature during the month of admission, USDA climate zone of the admitting hospital, patient age, patient sex, and hospital bed size. Estimated coefficients for each predictor variable were analyzed for significance using the Wald $\chi^{2}$ test. Regression coefficients were transformed to reflect the $\mathrm{RR}$ difference in response between the tested and fixed variable using the exponential transformation, $\mathrm{y}=\mathrm{e}^{\mathrm{x}}$. Correction for overdispersion with a weighting factor $\phi$ (Pearson $\chi^{2} /$ degrees of freedom) was used to scale parameter estimates and errors to conform to Poisson-distribution assumptions.

For each effect variable (SAH incidence and SAH in-hospital mortality incidence), separate models were constructed to study their relation with admission month or temperature/climate. Admission month regression models were unadjusted for other predictor variables, whereas temperature/climate zone models were adjusted for differences in age, sex, and hospital bed size (large versus medium and small). The primary sampling unit of the temperature/climate zone models was the NIS Hospital ID code, sorted by other included pre- 
Table 1: Distribution of SAH admissions, deaths, and hospital characteristics by USDA zone

\begin{tabular}{|c|c|c|c|c|c|c|}
\hline \multirow[b]{2}{*}{ Zone } & \multirow[b]{2}{*}{$\begin{array}{l}\text { Hospitals } \\
\qquad(n)\end{array}$} & \multirow[b]{2}{*}{$\begin{array}{c}\text { Admissions } \\
(n)\end{array}$} & \multirow[b]{2}{*}{$\begin{array}{l}\text { Deaths } \\
(n[\%])\end{array}$} & \multicolumn{3}{|c|}{ Hospital Characteristics by Admission Counts ${ }^{a}$} \\
\hline & & & & $\begin{array}{l}\text { Teaching } \\
(n[\%])^{\mathrm{b}}\end{array}$ & $\begin{array}{c}\text { Large } \\
(n[\%])^{c}\end{array}$ & $\begin{array}{l}\text { Urban } \\
(n[\%])^{\mathrm{d}}\end{array}$ \\
\hline 3 & 8 & 266 & $64(24.1)$ & $255(95.9)$ & $184(69.2)$ & $259(97.4)$ \\
\hline 4 & 118 & 1656 & $378(22.8)$ & $1219(73.6)$ & $1028(62.1)$ & $1458(88.0)$ \\
\hline 5 & 374 & 6967 & $1737(25.0)$ & $5079(72.9)$ & $4973(71.4)$ & $6339(90.9)$ \\
\hline 6 & 671 & 13,251 & $3429(25.9)$ & $10,416(78.6)$ & $10,385(78.4)$ & $12,653(95.5)$ \\
\hline 7 & 414 & 9829 & $2517(25.6)$ & $7619(77.5)$ & $7388(75.2)$ & $9338(95.0)$ \\
\hline 8 & 351 & 8677 & $2126(24.5)$ & $5356(61.7)$ & $6711(77.4)$ & $8328(96.0)$ \\
\hline 9 & 368 & 7971 & $2008(25.2)$ & 4598 (57.8) & 5633 (70.8) & 7769 (97.6) \\
\hline 10 & 131 & 4087 & $1013(24.8)$ & 2372 (58.0) & $3270(80.0)$ & 4061 (99.5) \\
\hline 11 & 16 & 192 & $57(29.7)$ & 106 (55.2) & $72(37.5)$ & $158(82.2)$ \\
\hline
\end{tabular}

${ }^{a}$ Among hospitals with recorded SAH admissions.

${ }^{\mathrm{b}}$ Compared with nonteaching hospitals.

${ }^{\circ}$ Compared with small and medium-sized hospitals.

${ }^{\mathrm{d}}$ Compared with rural hospital locations.
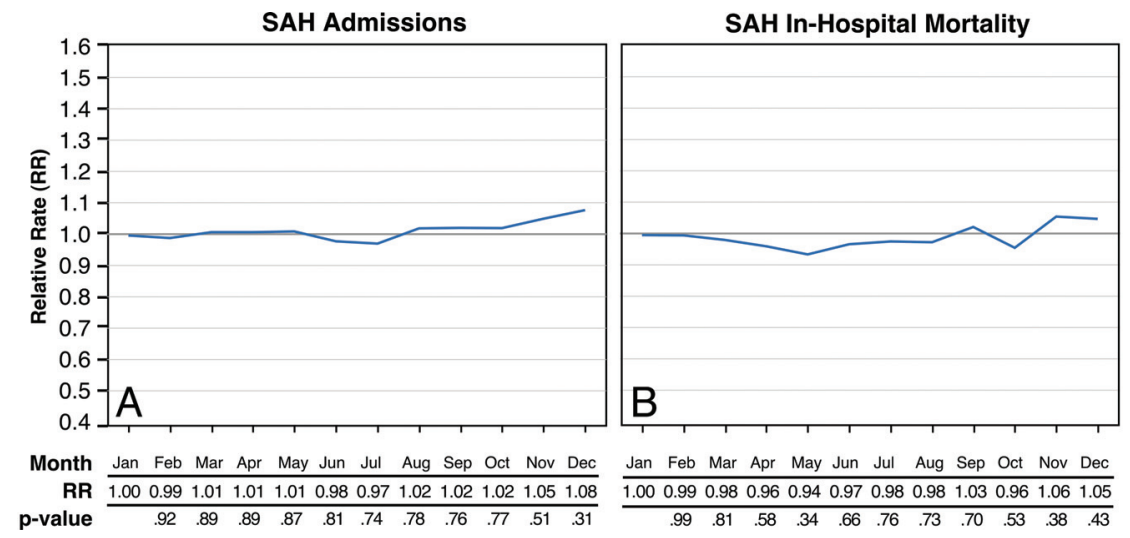

Fig 2. Monthly stratified relative rates of $(A)$ admission frequency and $(B)$ in-hospital mortality after admission for SAH. Relative rates of SAH admission and in-hospital mortality, stratified by month, were determined from regression models. RRs greater than 1.0 suggest that the event (admission or death) is more likely to occur relative to the reference month (Jan), whereas RRs less than 1.0 suggest the event is less likely to occur relative to the reference month. Significant differences relative to the reference month are represented by an asterisk $\left({ }^{*}\right)$.

dictor variables. The impact of clustered data on unequal sampling of the hospitals within the NIS on the observed variance of the effect variable was estimated with the REML method, using a randomeffects model assigned to each unique hospital identifier. The impact of colinearity of temperature and USDA zone on the model results was assessed using the VIF; values below 10 were suggestive of nonsignificant colinearity, while values above 10 were suggestive of significant colinearity.

\section{Results}

\section{Demographic Data}

From 2001 to 2008, the NIS recorded 52,896 cases of SAH from 57,663,486 total hospital admissions, representing $0.092 \%$ of all recorded hospitalizations. Among these nontraumatic SAH admissions, 13,272 (25.1\%) patients died during their initial hospitalization. In total, these SAH admissions were recorded by 2435 hospitals, spread across 20 states and representing USDA zones 3-11. The frequencies of SAH admissions, SAH in-hospital mortality, and characteristics of the hospitals responsible for SAH admissions, sorted by USDA climate zone, are shown in Table 1. Significant differences were seen across USDA zones with regard to the frequency of SAH deaths $\left(\chi^{2}=16.7, P=.0195\right)$, hospital location $\left(\chi^{2}=169.6, P<.0001\right)$, hospital bed size $\left(\chi^{2}=15.3, P<.0001\right)$, and hospital teaching status $\left(\chi^{2}=\right.$ 32.2, $P<.0001)$.

\section{Effect of Month on SAH Incidence Rates and In-Hospital Mortality Rates}

Regression analysis failed to demonstrate a significant relationship between SAH admission rates and month ([SAH frequency: Month] $\left.\chi^{2}=2.94, d f=11, P=.9914\right)$. These results, shown in Fig $2 A$, revealed insignificant month-to-month variation of between $2 \%-8 \%$ from January, the reference month held constant in the regression model. No significant increases in SAH rates were observed to suggest the presence of seasonal periodicity of SAH incidence.

Because in-hospital mortality presumably represents the most severe cases of SAH, we decided to investigate whether these events were uniquely associated with seasonal effects. Regression analysis used to study how SAH admission rates varied with month failed to demonstrate a significant effect ([SAH deaths: Month] $\chi^{2}=6.911, d f=11, P=.8062$ ). These results, shown in Fig $2 B$, revealed insignificant month-tomonth variation compared with the reference month of January. As before, no significant increase in SAH in-hospital mortality rates was observed in a pattern that might be suggestive of a seasonal effect. 
Table 2: Effects of climate, patient demographics, and hospital characteristics on the incidence of SAH and SAH-related mortality

SAH Incidence Model

$\left(\chi^{2}=3483, d f=20, P<.0001\right)$

\section{Variable}

Temperature stratum $(d f=6)^{c}$

USDA zone $(d f=8)^{c}$

Age $(d f=1)^{\mathrm{d}}$

Female gender $(d f=1)^{\mathrm{e}}$

Teaching hospitals $(d f=1)^{f}$

Large hospital bed size $(d f=1)^{g}$

Urban location $(d f=1)^{\mathrm{h}}$

andicates significant effects.

b RR with 95\% confidence interval as defined in Materials and Methods.

c See Materials and Methods for a description of stratifications and groupings.

${ }^{\mathrm{d}}$ Change in RR for every 10-year increase in age.

e Compared to males.

${ }^{\dagger}$ Compared with nonteaching hospitals.

${ }^{g}$ Compared with small and medium-sized hospitals.

${ }^{\mathrm{h}}$ Compared with rural hospital locations.
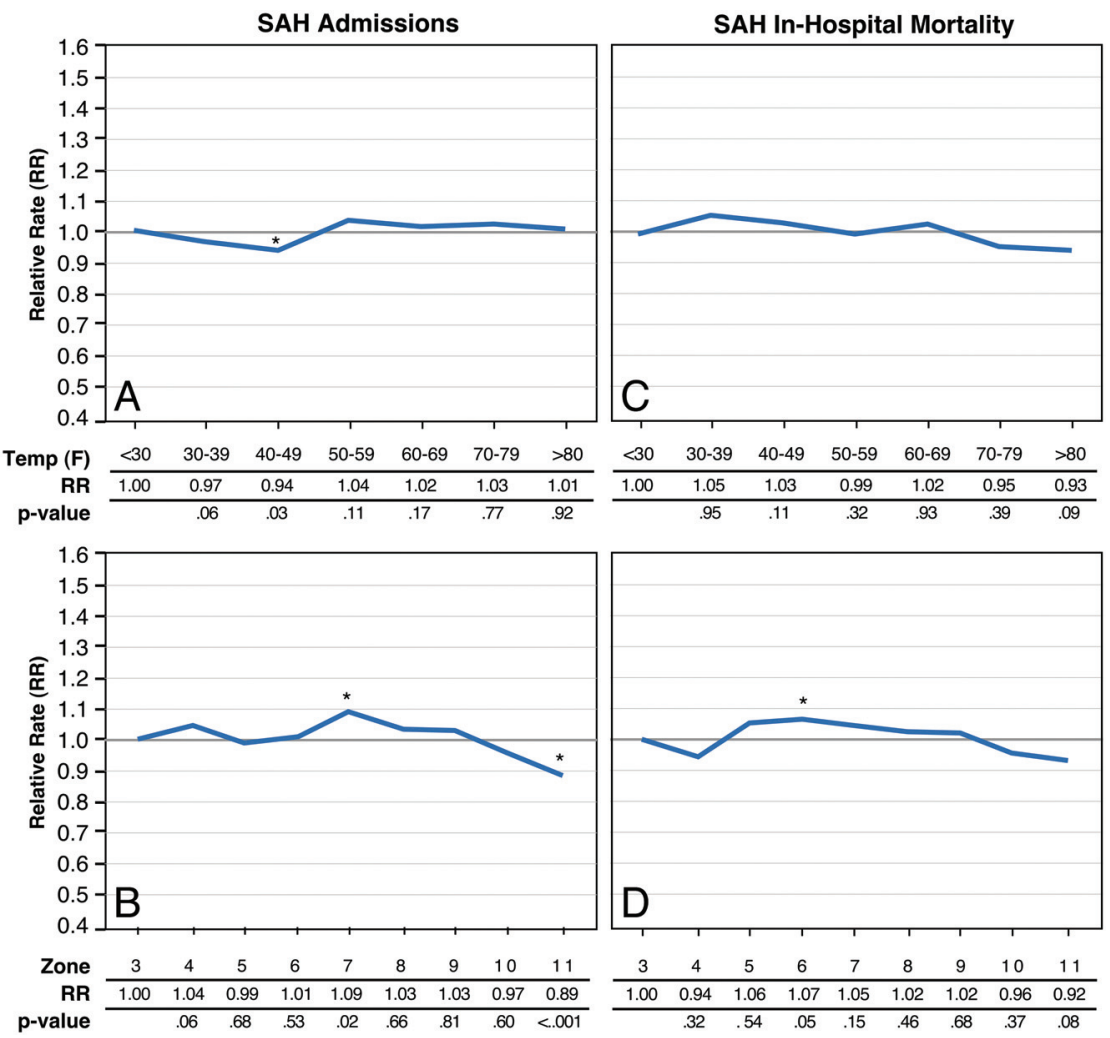

Fig 3. Temperature $(A, C)$ and climate $(B, D)$ stratified relative rates for $(A, B)$ admission frequency and $(D, E)$ in-hospital mortality after admission for $S A H$. Relative rates of $S A H$ admission and in-hospital mortality, stratified by temperature and climate, were determined from regression models. RRs greater than 1.0 suggest that the event (admission or death) is more likely to occur relative to the reference variable (temperature strata $\left[<30^{\circ} \mathrm{F}\right.$ ]; climate strata [USDA zone 3]), whereas RRs less than 1.0 suggest the event is less likely to occur relative to the reference variable. Significant differences relative to the reference temperature stratum or zone are represented by an asterisk $\left(^{*}\right)$.

\section{Multivariate Regression Model Results: The Effect of Temperature and Climate on SAH Incidence and In-Hospital Mortality Rates}

After adjusting for other predictor variables, multivariate regression analysis showed that the incidence of $\mathrm{SAH}$ was insensitive to average temperature (Table $2, P=.1453$; Fig $3 A$ ) and only modestly affected by climate region (Table $2, P=.0009$; Fig 3C). Relative to zone 3, the frequency of SAH admissions was significantly lower in zone 11 and significantly higher in zone 7 , and these differences amount to a $20 \%$ difference in the SAH incidence rate. Admission frequencies were not significantly different between other climate zones. Female sex, large hospital size, and urban hospital location were associated with increased SAH (Table 2). Hospital teaching status was the most strongly predictive variable of SAH admission in this model (Table 2; RR $=1.25(1.20-1.32), P<.0001)$. Intraclass correlations of hospital admission data were found to contribute modestly to the total observed variance of SAH admissions $(9.1 \%)$. Minimal predictor variable colinearity was observed in this model (VIF range, 1.01-5.34).

Similar multivariate analysis revealed that the in-hospital mortality rate after admission for SAH was not affected by temperature (Table 2, $P=.3005$; Fig 3B) or climate zone (Table 2, $P=.0863$; Fig $3 D$ ). Increasing age and nonteaching 
hospitals were both strongly predictive of greater SAH deaths (Table 2; age [per 10-year increment]: $\mathrm{RR}=1.18(1.16-1.19)$, $P<.0001$; teaching hospitals: $\mathrm{RR}=0.90(0.85-0.97), P<$ $.0001)$. No other predictor variables were significantly associated with SAH mortality. In this model, intraclass correlation of hospital admission data were found to contribute only minimally to the total observed variance of SAH mortality (4.4\%). Minimal predictor variable colinearity was observed in the SAH death model (VIF range, 1.00-5.38).

\section{Discussion}

The results of this retrospective review of the Nationwide Inpatient Sample provide robust evidence for the lack of seasonal variation on admission and in-hospital mortality rates for spontaneous SAH. Our data suggest that climate, but not average monthly temperature, may weakly play a role in the frequency of SAH incidence, yet sporadically, without strong association or trends with other meteorologic variables. Finally, in-hospital mortality rates after SAH admission did not significantly vary with changes in average monthly temperature or climate zone.

Our data reveal that SAH incidence frequencies, after adjustment for patient demographics and hospital characteristics, remain weakly associated with climate zone. However, these changes represent fairly modest deviations in the observed rates of SAH incidence and mortality across the sampled US population. Further, these deviations in the data do not manifest in a systematic pattern that would be expected if SAH had a meaningful association with climate. Such a pattern would be expected to present as a linear trend with climate zone. The origins of these zone-dependent effects, unfortunately, are beyond the scope of this study. Given the heterogeneous demographic distributions across the United States, it remains possible that the observed zone-dependent effect results from demographic nonuniformity in 1 or more SAH risk factors. ${ }^{32,49}$ Finally, these zone effects could be a result of biased sampling of hospital types intrinsic to the NIS dataset.

Recently, Cowperthwaite and Burnett ${ }^{39}$ also failed to identify an influence of short- and long-term variation in weather, including daily temperature, barometric pressure, and humidity, with SAH volumes within a group of 155 hospitals comprising 7758 SAH admissions. Our current analysis complements these previous findings by generalizing them to a larger dataset obtained from a broader range of climates and temperatures found within the complex geographic landscape of the United States.

The cause for the lack of consensus among previous studies is probably a function of several phenomena. First, it has been suggested that reports of seasonal patterns are simply a manifestation of referral bias. ${ }^{37}$ Second, several of the well-established risk factors for $\mathrm{SAH}$, including hypertension, tobacco use, and ethnicity, are variably expressed in different regions of the United States, and such factors could confound the results of studies conducted in more isolated geographic zones. ${ }^{49}$ Third, the conclusions of many previous studies were drawn from significantly smaller sample sizes for shorter periods of time, and these sampling limits could easily introduce bias, making it difficult to assign significance to changes observed in SAH frequencies. Fourth, as pointed out by Cow- perthwaite and Burnett, ${ }^{37}$ many weather variables are highly colinear and thus it is very difficult to extricate their individual effects without confounding the results. ${ }^{39}$

This study has several limitations related to the utilization of an extremely large retrospective data base with a predefined data structure. First, as the NIS is insensitive to mortality events taking place before hospital admission, patient mortality in the home and in the emergency department setting are not captured in our analysis. Second, our retrospective study design was limited to the resolution of the dataset, and thus daily changes in weather patterns were beyond the predefined sensitivity of the dataset. Third, coding errors are a well-established limitation of such data sources and have been shown to exist within the NIS data. ${ }^{50}$ Fortunately, it has been suggested that such errors follow a random distribution and do not manifest in a systemic fashion, and thus are unlikely to impact the statistical results because they are diluted within the enormous size of the NIS dataset. Finally, the predefined data limits of the NIS prevent us from ascertaining causal relationships and outcomes after hospitalization that may better explain observed effects in statistical regression models.

\section{Conclusions}

Our findings suggest that season, temperature, and climate are not significant risk factors for the development of SAH or SAH-related mortality, as they fail to demonstrate periodic or linear trends with these proposed risk factors. A few sporadic differences in SAH incidence and mortality frequencies were observed, but these differences were small and are likely explained by regional demographic differences and not by meteorologic or climate-related effects.

Disclosures: David F. Kallmes—UNRELATED: Grants/Grants Pending: NFocus, ${ }^{*}$ Sequent, ${ }^{*}$ Penumbra, ${ }^{*}$ MicroVention, ${ }^{*}$ eV3*; Payment for Development of Educational Presentations: CareFusion, ${ }^{*}$ eV3. ${ }^{*}$ Harry J Cloft—UNRELATED: Consultancy: Medtronic, Comments: Serve on the Data Safety and Monitoring Board for the KAVIAR study (Kyphoplasty And Vertebroplasty In the Augmentation and Restoration of Vertebral Body Compression Fractures); Grants/Grants Pending: Cordis, ${ }^{*}$ Mindframe. ${ }^{*}$ ( Money paid to institution.)

\section{Acknowledgments}

The authors would like to thank Rickey Carter, $\mathrm{PhD}$, for statistical consultation and assistance.

\section{References}

1. Bederson JB, Connolly ES Jr, Batjer HH, et al. Guidelines for the management of aneurysmal subarachnoid hemorrhage: a statement for healthcare professionals from a special writing group of the Stroke Council, American Heart Association. Stroke 2009;40:994-1025

2. Suarez JI, Tarr RW, Selman WR. Aneurysmal subarachnoid hemorrhage. N Engl J Med 2006;354:387-96

3. van der Bilt IA, Hasan D, Vandertop WP, et al. Impact of cardiac complications on outcome after aneurysmal subarachnoid hemorrhage: a meta-analysis. Neurology 2009;72:635-42

4. Qureshi AI, Suri MF, Yahia AM, et al. Risk factors for subarachnoid hemorrhage. Neurosurgery 2001;49:607-12

5. Broderick JP, Viscoli CM, Brott T, et al. Major risk factors for aneurysmal subarachnoid hemorrhage in the young are modifiable. Stroke 2003;34: 1375-81

6. Aring CD, Merritt HH. Differential diagnosis between cerebral hemorrhage and cerebral thrombosis. Arch Intern Med 1935;56:435-56

7. Takahashi E, Sasaki N, Takeda J, et al. The geographic distribution of cerebral hemorrhage and hypertension in Japan. Hum Biol 1957;29:139-66

8. Ramirez-Lassepas M, Haus E, Lakatua DJ, et al. Seasonal (circannual) periodicity of spontaneous intracerebral hemorrhage in Minnesota. Ann Neurol 1980;8:539-41 
9. Haberman S, Capildeo R, Rose FC. The seasonal variation in mortality from cerebrovascular disease. Neurol Sci 1981;52:25-36

10. Sobel E, Zhang ZX, Alter M, et al. Stroke in the Lehigh Valley: seasonal variation in incidence rates. Stroke 1987;18:38-42

11. Biller J, Jones MP, Bruno A, et al. Seasonal variation of stroke: does it exist? Neuroepidemiology 1988;7:89-98

12. Shinkawa A, Ueda $\mathrm{K}$, Hasuo $\mathrm{Y}$, et al. Seasonal variation in stroke incidence in Hisayama, Japan. Stroke 1990;21:1262-67

13. Capon A, Demeurisse G, Zheng L. Seasonal variation of cerebral hemorrhage in 236 consecutive cases in Brussels. Stroke 1992;23:24-27

14. Franke CL, van Swieten JC, van Gign J. Circadian and seasonal variation in the incidence of intracerebral hemorrhage. Cerebrovasc Dis 1992;2:44-46

15. Ricci S, Celani MG, Vitali R, et al. Diurnal and seasonal variations in the occurrence of stroke: a community-based study. Neuroepidemiology 1992;11: 59-64

16. Chyatte D, Chen TL, Bronstein K, et al. Seasonal fluctuation in the incidence of intracranial aneurysm rupture and its relationship to changing climatic conditions. J Neurosurg 1994;81:525-30

17. Caplan LR, Neely S, Gorelick P. Cold-related intracerebral hemorrhage. Arch Neurol 1984;41:227

18. Langmayr JJ, Obwegeser A, Ortler M. Seasonal rupture of aneurysms. J Neurosurg 1995;83:182-83

19. Landers AT, Narotam PK, Govender ST, et al. The effect of changes in barometric pressure on the risk of rupture of intracranial aneurysms. Br J Neurosurg 1997;11:191-95

20. Inagawa $\mathrm{T}$, Takechi $\mathrm{A}$, Yahara $\mathrm{K}$, et al. Primary intracerebral and aneurysmal subarachnoid hemorrhage in Izumo City, Japan Part I: incidence and seasonal and diurnal variations. J Neurosurg 2000;93:958-66

21. Buxton N, Liu C, Dasic D, et al. Relationship of aneurysmal subarachnoid hemorrhage to changes in atmospheric pressure: results of a prospective study. J Neurosurg 2001;95:391-92

22. Nyquist PA, Brown RD, Wiebers DO, et al. Circadian and seasonal occurrence of subarachnoid and intracerebral hemorrhage. Neurology 2001;56:190-93

23. Setzer M, Beck J, Hermann E, et al. The influence of barometric pressure changes and standard meteorological variables on the occurrence and clinical features of subarachnoid hemorrhage. Surg Neurol 2007;67:264-72

24. Abe T, Ohde S, Ishimatsu S, et al. Effects of meteorological factors on the onset of subarachnoid hemorrhage: a time-series analysis. J Clin Neurosci 2008;15: 1005-10

25. Beseoglu K, Hänggi D, Stummer W, et al. Dependence of subarachnoid hemorrhage on climate conditions: a systematic meteorological analysis from the Dusseldorf metropolitan area. Neurosurgery 2008;62:1033-38

26. Kleinpeter G, Schatzer R, Bock F. Is blood pressure really a trigger for the circadian rhythm of subarachnoid hemorrhage? Stroke 1995;26:1805-10

27. Vermeer SE, Rinkel GJ, Algra A. Circadian fluctuations in onset of subarachnoid hemorrhage. New data on aneurysmal and perimesencephalic hemorrhage and a systematic review. Stroke 1997;28:805-08

28. Muller JE, Tofler GH, Stone PH. Circadian variation and triggers of onset of acute cardiovascular disease. Circulation 1989;79:733-43

29. Elliott WJ. Circadian variation in the timing of stroke onset: a meta-analysis. Stroke 1998;29:992-96

30. Wroe SJ, Sandercock P, Bamford J, et al. Diurnal variation in incidence of stroke: Oxfordshire Community Stroke Project. BMJ 1992;304:155-57
31. Sloan MA, Price TR, Foulkes MA, et al. Circadian rhythmicity of stroke onset. Intracerebral and subarachnoid hemorrhage. Stroke 1992;23:1420-26

32. Albert CM, Mittleman MA, Chae CU, et al. Triggering of sudden death from cardiac causes by vigorous exertion. $N$ Engl J Med 2000;343:1355-61

33. Giaconi S, Ghione S, Palombo C, et al. Seasonal influences on blood pressure in high normal to mild hypertensive range. Hypertension 1989;14:22-27

34. Lejeune JP, Vinchon M, Amouyel P, et al. Association of occurrence of aneurysmal bleeding with meteorologic variations in the north of France. Stroke 1994;25:338-41

35. Field TS, Zhu H, Tarrant M, et al. Relationship between supra-annual trends in influenza rates and stroke occurrence. Neuroepidemiology 2004;23:228-35

36. Piñol-Ripoll G, de la Puerta I, Santos S, et al. Chronic bronchitis and acute infections as new risk factors for ischemic stroke and the lack of protection offered by the influenza vaccination. Cerebrovasc Dis 2008;26:339-47

37. Rothwell PM, Wroe SJ, Slattery J, et al. Is stroke incidence related to season or temperature? The Oxfordshire Community Stroke Project. Lancet 1996;347: $934-36$

38. Woodhouse PR, Khaw K, Plummer M, et al. Seasonal variations of plasma fibrinogen and factor VII activity in the elderly: winter infections and death from cardiovascular disease. Lancet 1994;343:435-39

39. Cowperthwaite MC, Burnett MG. The association between weather and spontaneous subarachnoid hemorrhage: an analysis of 155 US hospitals. Neurosurgery 2011;68:132-38, discussion 138-39

40. Schievink WI, Wijdicks EF, Meyer FB, et al. Seasons, snow, and subarachnoid hemorrhage: lack of association in Rochester, Minnesota. J Neurosurg 1995;82: 912-13 934-36, 1996

41. Oberg AO, Ferguson JF, McIntyre LM, et al. Incidence of stroke and season evidence for an association. Am J Epidemiol 2000;152:558-64

42. Field TS, Hill MD. Weather, Chinook, and stroke occurrence. Stroke 2002;33: 1751-57

43. Healthcare Cost and Utilization Project (HCUP) 1998-2004. HCUP database. Rockville, MD: Agency for Healthcare Research and Quality. Available at: http:// www.hcup-us.ahrq.gov/databases.jsp. Accessed January 10, 2011

44. Healthcare Cost and Utilization Project (HCUP) 1998-2004. Nationwide Inpatient Sample description of data elements. Rockville, MD: Agency fo Healthcare Research and Quality. Available at: http://www.hcup-us.ahrq.gov/ $\mathrm{db} /$ nation/nis/nisdde.jsp. Accessed August 27, 2011

45. National Oceanic and Atmospheric Administration. NOAA climatology database. Available at: http://lwf.ncdc.noaa.gov/oa/climate/climatedata.html. Accessed July 12, 2011

46. United States Department of Agriculture. USDA plant hardiness zone maps. Available at: http://www.usna.usda.gov/Hardzone/hrdzon2.html, and http:// www.usna.usda.gov/Hardzone/ushzmap.html. Accessed January 10, 2011

47. United States Department of Agriculture. Interactive plant maps. Available at: http://www.plantmaps.com/usda_hardiness_zone_map.php. Accessed August 27,2011

48. American Horticultural Society. Heat zone finder. Available at: http://www. ahs.org/publications/heat_zone_finder.htm. Accessed August 27, 2011

49. Casper ML, Barnett E, Williams GI, et al. Atlas of Stroke Mortality: Racial, Ethnic, and Geographic Disparities in the United States. Atlanta: U.S. Department of Health and Human Services, CDC; 2003

50. Berthelsen CL. Evaluation of coding data quality of the HCUP National Inpatient Sample. Top Health Inf Manage 2000;21:10-23 